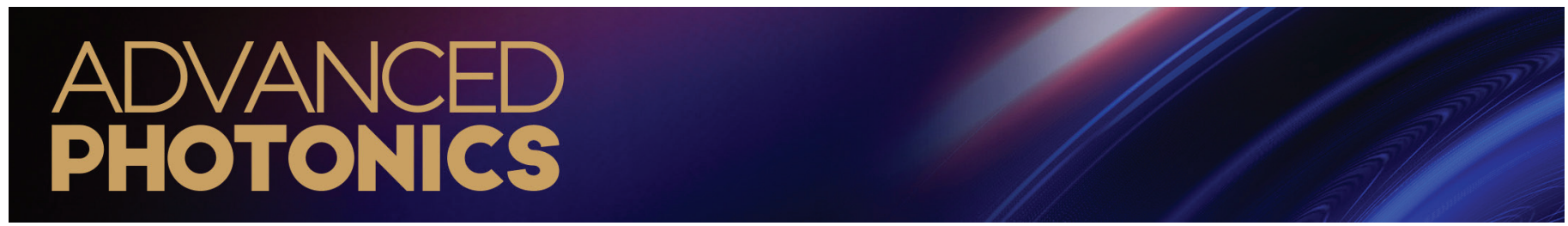

\title{
Plasma-grating-induced breakdown spectroscopy
}

\author{
Mengyun $\mathrm{Hu}^{\mathrm{a}, \mathrm{b}}$ Junsong Peng $\odot{ }^{\mathrm{a}, \mathrm{b}}$ Sheng Niu, ${ }^{\mathrm{a}}$ and Heping Zeng ${ }^{\mathrm{a}, \mathrm{b}, \mathrm{c}, *}$ \\ ${ }^{a}$ East China Normal University, State Key Laboratory of Precision Spectroscopy, Shanghai, China \\ ${ }^{\mathrm{b}}$ Chongqing Institute of East China Normal University, Chongqing, China \\ 'Jinan Institute of Quantum Technology, Jinan, China
}

\begin{abstract}
Laser-induced breakdown spectroscopy (LIBS) is a useful tool for determination of elements in solids, liquids, and gases. For nanosecond LIBS (ns-LIBS), the plasma shielding effect limits its reproducibility, repeatability, and signal-to-noise ratios. Although femtosecond laser filament induced breakdown spectroscopy (FIBS) has no plasma shielding effects, the power density clamping inside the filaments limits the measurement sensitivity. We propose and demonstrate plasma-grating-induced breakdown spectroscopy (GIBS). The technique relies on a plasma excitation source-a plasma grating generated by the interference of two noncollinear femtosecond filaments. We demonstrate that GIBS can overcome the limitations of standard techniques such as ns-LIBS and FIBS. Signal intensity enhancement with GIBS is observed to be greater than 3 times that of FIBS. The matrix effect is also significantly reduced with GIBS, by virtue of the high power and electron density of the plasma grating, demonstrating great potential for analyzing samples with complex matrix.
\end{abstract}

Keywords: femtosecond filament; plasma grating induced breakdown spectroscopy; high power and electron density; enhancement; matrix effect.

Received May 9, 2020; accepted for publication Sep. 22, 2020; published online Oct. 21, 2020.

(C) The Authors. Published by SPIE and CLP under a Creative Commons Attribution 4.0 Unported License. Distribution or reproduction of this work in whole or in part requires full attribution of the original publication, including its DOI.

[DOI: 10.1117/1.AP.2.6.065001]

\section{Introduction}

Laser-induced breakdown spectroscopy (LIBS) is one of the most effective tools used to study the interaction between lasers and matter. Compared with other techniques, like atomic absorption spectroscopy and inductively coupled plasma optical emission spectroscopy, LIBS has some unique advantages including the capacity of direct analysis without a complex pretreatment of the sample, rapid response, and multiple elemental analysis ability. LIBS is suitable for special requirements of practical solid, ${ }^{1-4}$ liquid,,$^{5-8}$ and gas analyses. ${ }^{9,10}$

For traditional LIBS systems, nanosecond (ns) pulse lasers are widely used. But due to some adverse impacts like laser emission intensity, long pulse duration, and plasma shielding effect, ${ }^{11}$ the ns-LIBS has drawbacks, including low reproducibility and repeatability, low signal-to-noise ratios, and difficult molecular measurements. In contrast, the pulse duration of femtosecond lasers is much shorter, such that there is no plasma shielding effect and the interaction time of laser and matter is very short. Therefore, signal-to-background ratios and

*Address all correspondence to Heping Zeng, E-mail: hpzeng@ phy.ecnu.edu.cn resolutions of spectral lines are higher. ${ }^{12,13}$ Meanwhile, because of the high power density, materials could be ionized and dissociated quickly, and thus the ions and molecular fragments could be stimulated effectively. ${ }^{14-16}$

Filament-induced breakdown spectroscopy (FIBS) combines the LIBS technique and a femtosecond laser filament. ${ }^{17}$ When a high-intensity femtosecond laser pulse transmits in the air, due to the dynamic balance between the self-focusing of the Kerr effect and the defocusing of the plasma formed by multiphoton ionization, a long and stable laser plasma channel forms, i.e., a femtosecond (fs) laser filament. ${ }^{18-20}$ In addition to the advantages of the fs laser pulse, FIBS can overcome the diffraction limit and deliver high laser intensities at remote locations. ${ }^{17}$ Systematical comparative investigations on LIBS using ns and fs pulses, as well as filaments under different conditions, were recently reported. ${ }^{21,22}$ The laser intensity clamping effect is a limitation of FIBS, ${ }^{23-26}$ as the power and electron densities are saturated when the laser energy increases. This imposes a limitation on the sensitivity improvement. The interaction of multiple femtosecond filaments could generate plasma grating $\mathrm{s}^{27-32}$ and can break through the clamped intensity limit for free electron generation and acceleration. ${ }^{33}$ 
In this work, we propose and demonstrate the plasmagrating-induced breakdown spectroscopy (GIBS) technique. The plasma grating is generated by two noncollinear femtosecond filaments. GIBS is proven to be useful to overcome the drawbacks of ns-LIBS, fs-LIBS, and FIBS. With GIBS, the signal intensity enhancement is greater than 3 times that obtained by FIBS with the same initial pulse. The plasma evolution is studied, and the lifetime of plasma-grating-induced plasma is found to be about twice that of filament-induced plasma. Due to the absence of plasma shielding effects, the high power, and electron density of the femtosecond plasma grating, materials could be ionized and dissociated in a more uniform manner so that the matrix effect is reduced and quantitative analyses are feasible.

\section{Experimental Details}

\subsection{Experimental Setup}

Our experimental schematic is shown in Fig. 1. Intense fs pulses were generated from a Ti:sapphire regenerative laser (Coherent) operating at $800 \mathrm{~nm}$ with a repetition rate of $1 \mathrm{kHz}$, a pulse duration of $50 \mathrm{fs}$, and a pulse energy up to $6 \mathrm{~mJ}$. The fs laser pulses were split into two beams with almost the same energy, which were simultaneously focused by a convex lens $(f=100 \mathrm{~mm})$ to produce two noncollinearly crossed filaments in air. The crossing angle $\theta$ of two filaments is $6.1 \mathrm{deg}$, resulting in spatial period $\Lambda$ of $7.5 \mu \mathrm{m}$ determined by $\Lambda=\lambda / 2 \sin (\theta / 2) .{ }^{34}$ The plasma grating was generated in the intersecting region of the two filaments and then ablated sample pellets to induce the plasma emission. If one beam is blocked, this would become the fs filament-induced plasma. Sample pellets were mounted on a motorized rotation displacement stage. The plasma emission was collected by a focus lens into a piece of optical fiber with a diameter of $50 \mu \mathrm{m}$. The fiber delivered the plasma emission into an Echelle spectrometer (Mechelle 5000, Andor Technology) equipped with an intensified charge coupled device (ICCD; iStar, Andor Technology). The acquisition mode was a single scan and the ICCD worked in the integrate-on-chip mode with gate delay of $4 \mathrm{~ns}$ and gate width of $100 \mathrm{~ns}$.

\subsection{Sample Preparation}

Five standard reference soil samples (GSS-08, GSS-04, GSS03, GSS-20, and GSF-03) were used for the present study. All soil samples were ground carefully in a mortar to become homogeneous. Then $0.5 \mathrm{~g}$ of the obtained mixture powder was

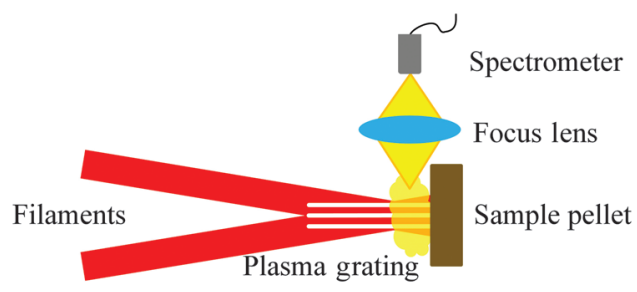

Fig. 1 Experimental schematic. Top view of the noncollinear interaction area where two filaments interfere and create a plasma grating, with a focus lens collecting the plasma emission into the spectrometer from the side direction after plasma grating ablating the sample pellet. filled into a steel dye of $13 \mathrm{~mm}$ diameter. A hydraulic press was applied on the powder to obtain a pellet. The pressure of $4 \mathrm{MPa}$ and time of $1 \mathrm{~min}$ were used for all pellets. Prepared pellets were then put on the motorized stage so that fresh materials could be irradiated and ablated continuously.

\section{Results and Discussion}

\subsection{Signal Enhancement of GIBS}

Plasma gratings are generated due to the nonlinear interaction of two filaments. ${ }^{30,32}$ The power density and electron density are higher than a single fs filament with the same pulse energy due to the constructive interference in the two-beam overlapping area. In this section, the exposure time of the spectrometer is set as $0.05 \mathrm{~s}$, considering the pulse interval of $0.001 \mathrm{~s}$ of the $1 \mathrm{kHz}$ repetitive femtosecond pulses, which means that each spectrum obtained under the integrate-on-chip mode is accumulated with 50 shots. Soil sample GSS-08 was used here. Figure 2(a) shows the relationship between the intensity of the Si $288.2 \mathrm{~nm}$ spectral line and the pulse energy. In Fig. 2(a), red circled points represent the results obtained by the FIBS. It can be seen that as the pulse energy increases, the signal intensity of Si tends to be saturated due to power clamping of the fs filament. Black squared points correspond to results measured by the GIBS. The signal intensity of the Si line obtained by the GIBS is about 3 times higher than that obtained by the FIBS using the same pulse energy. The two laser pulses used to form the plasma grating were equally split from the same laser pulse forming a single fs filament. The enhancement shown in Fig. 2(a) is because the interference of two filaments breaks through the clamped power of a single filament, so that the electron density inside the plasma channel is higher. For a single filament, the typical electron density is around $10^{17} \mathrm{~cm}^{-3}$, whereas for the plasma grating, the electron density is determined to be more than $10^{18} \mathrm{~cm}^{-3} \cdot{ }^{31}$ As a result, inside the plasma grating, the collision and acceleration of electrons are more intense. Some accelerated electrons trigger impact ionization of molecules and more electrons are generated, giving rise to the generation of stronger plasma emission. Figure 2(b) exhibits the influence of polarizations of the two synchronous pulses. If the polarizations are parallel, the signal intensity is about twice as high as that with perpendicular polarizations. This illustrates that the interference of two filaments with the same polarization generates a stronger signal than the simple superposition of two filaments with different polarizations.

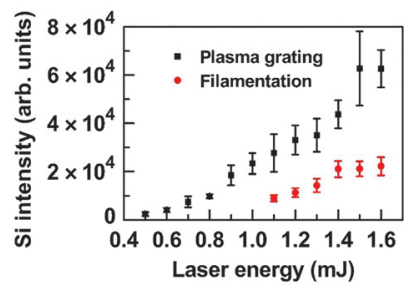

(a)

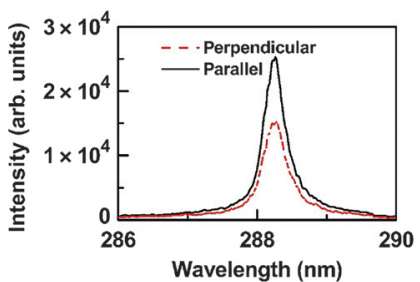

(b)
Fig. 2 (a) Intensity of the Si $288.2 \mathrm{~nm}$ line as a function of the laser pulse energy detected with the FIBS and GIBS systems. (b) Intensity of the Si $288.2 \mathrm{~nm}$ line obtained by interaction of two beams with different polarizations. 


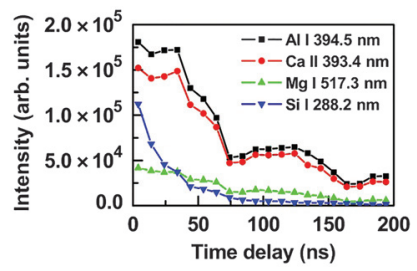

(a)

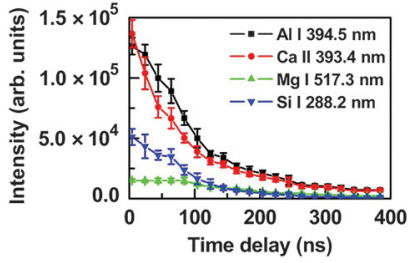

(b)
Fig. 3 Time evolution for intensities of four spectral lines (Al $394.5 \mathrm{~nm}, \mathrm{Ca} 393.4 \mathrm{~nm}, \mathrm{Mg} 517.3 \mathrm{~nm}$, and Si $288.2 \mathrm{~nm}$ ) detected with (a) an FIBS and (b) a GIBS system.

\subsection{Time Evolution of Induced Plasma}

Based on the results described above, in order to better understand the signal enhancement of the GIBS, we analyzed the time evolution of the plasma emission from filament and plasma grating ablation, respectively. Soil sample GSS-08 was used. The time evolutions of the intensities of four spectral lines (Al $394.5 \mathrm{~nm}, \mathrm{Ca} 393.4 \mathrm{~nm}, \mathrm{Mg} 517.3 \mathrm{~nm}$, and $\mathrm{Si}$ $288.2 \mathrm{~nm}$ ) are shown in Fig. 3 depicting the evolution of the corresponding plasma emission. Figures 3(a) and 3(b) depict the time evolution of the spectral line intensities under the filament and plasma grating conditions. The lifetime is the time taken for the intensity to decay to $1 / 3$ of its initial value. In Fig. 3(a), the average lifetime of plasma calculated from four selected spectral lines is $64 \mathrm{~ns}$, whereas in Fig. 3(b) it is $132 \mathrm{~ns}$. Nearly twice extension of the plasma lifetime demonstrates that with the GIBS, the fluorescence emission of the sample plasma lasts longer and within the same collection time, the signal intensity is accumulated to a higher level. Also, plasma emission is not the direct result of the laser ablation and excitation, which should be due to a secondary process like electron collisional excitation. ${ }^{33}$ This means that a longer plasma lifetime corresponds to a higher electron density in the plasma channel, which is also consistent with the results presented in Sec. 3.1.

\subsection{Reduction of Matrix Effect}

In order to study the matrix effect, soil samples doped with a $\mathrm{Cr}$ element of different concentrations were used as target samples. The target elemental concentrations of these soil samples are given in Table 1. We prepared soil sample powders with $\mathrm{Cr}$ concentrations ranging from 68 to $4370 \mathrm{ppm}$.

Table 1 Element concentrations of soil samples in the experiment.

\begin{tabular}{lccccc} 
Sample & \multicolumn{5}{c}{ Element concentration } \\
\cline { 2 - 6 } name & $\mathrm{SiO}_{2}(\%)$ & $\mathrm{Al}_{2} \mathrm{O}_{3}(\%)$ & $\mathrm{CaO}(\%)$ & $\mathrm{MgO}(\%)$ & $\mathrm{Cr}(\mathrm{ppm})$ \\
\hline GSF-03 & $\mathrm{N} . \mathrm{A}$. & $\mathrm{N} . \mathrm{A}$. & 1.06 & $\mathrm{~N} . \mathrm{A}$. & 104 \\
GSS-03 & 74.72 & 12.24 & 1.27 & 0.58 & 32 \\
GSS-04 & 50.95 & 23.45 & 0.26 & 0.49 & 370 \\
GSS-08 & 58.61 & 11.92 & 8.27 & 2.38 & 68 \\
GSS-20 & 47.28 & 10.39 & 6.48 & 2.98 & 43 \\
\hline
\end{tabular}

Note: N.A., not available.

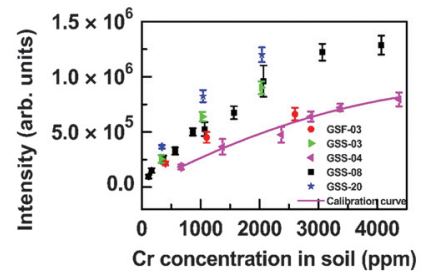

(a)

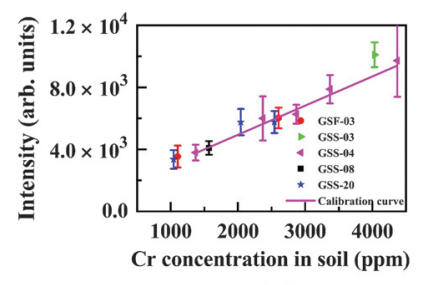

(c)

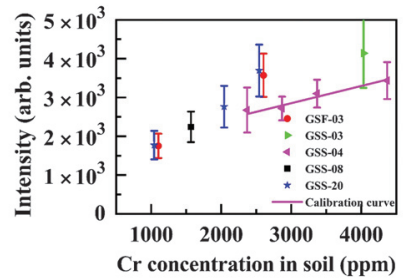

(b)

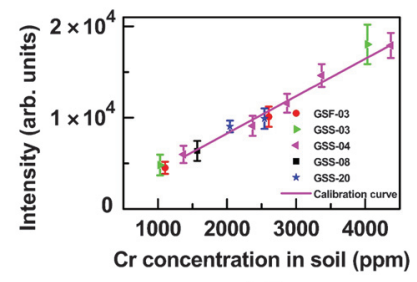

(d)
Fig. 4 Intensity of the $\mathrm{Cr} 425.43 \mathrm{~nm}$ line as a function of the prepared $\mathrm{Cr}$ concentration detected with (a) ns-LIBS, (b) fs-LIBS, (c) FIBS, and (d) GIBS systems.

First, we used the previous ns-LIBS experimental system described in Refs. 8 and 34 to detect these soil samples. Figure 4(a) depicts the average intensity of the $\mathrm{Cr} 425.43 \mathrm{~nm}$ line as a function of the $\mathrm{Cr}$ concentrations. Error bars correspond to the standard deviations of the six replicate measurements. Soil sample GSS-04 was used as the reference sample to obtain a calibration curve that was fitted with parabola (pink solid line) with a regression coefficient $R^{2}$ of $99.0 \%$. As shown in Fig. 4(a), the data points obtained from other soil samples exhibit a large dispersion around the calibration curve, clearly showing a significant matrix effect among these samples.

In order to quantitatively assess the matrix effect among different samples, we calculated the average relative error of prediction (REP) for other soil samples with respect to the calibration curve of $\mathrm{S} 3$, as defined by

$\operatorname{REP}(\%)=\frac{100}{N} \sum_{i=1}^{N}\left|\frac{I_{p}-I_{i}}{I_{i}}\right|$

where $N$ is the number of different concentrations prepared for a validation sample, $I_{i}$ is the measured line intensity of $\mathrm{Cr}$, and $I_{p}$ is the corresponding predicted signal intensity retrieved from the calibration curve with the prepared concentration. The value of REP corresponds to the relative deviation between the actual measured intensity and the predicted intensity, which means the accuracy of predicted intensity calculated from calibration curves with known elemental concentrations, as well as that of the predicted concentration obtained from calibration curves with the measured signal intensity. The obtained REPs for all the validation samples range from $31.2 \%$ to $66.4 \%$, which are apparently larger than the standard deviations of data. It is obvious that the calibration curve established with the $\mathrm{S} 3$ sample cannot be used to correctly predict the chromium concentration for most of the validation samples because of the matrix effect.

Figures 4(b)-4(d) show the relationship between the $\mathrm{Cr}$ spectral line intensity and concentrations from different soil samples ablated by the fs pulse (pulse energy $0.3 \mathrm{~mJ}$ ), fs filament (pulse energy $1.6 \mathrm{~mJ}$ ), and fs plasma grating (two pulses with total energy $1.6 \mathrm{~mJ}$ ), respectively. Error bars for each data point 
correspond to the standard deviations of 20 replicated measurements. The dispersion of data points is much reduced compared to that shown in Fig. 4(a), which means the matrix effect has apparently been reduced. This should be due to the higher power density of the fs pulse and the more absolute ablation of samples. From Figs. 4(c) and 4(d), we can see that data points of different soil samples exhibit good linear distributions. In fact, the data in both Figs. 4(c) and 4(d) can be linearly fitted with regression coefficients of $R^{2}=95.5 \%$ and $98.0 \%$, respectively. The linear calibration curves established with different soil samples can be used to correctly predict the elemental concentrations of other soil samples detected with the same procedure.

For ns-LIBS, due to long pulse durations, there is a plasma shielding effect, ${ }^{11}$ which refers to the fact that laser-induced plasma absorbs part of incident laser energy and prevents the efficient coupling between laser and matter, resulting in a low utilization rate of laser energy. For different samples, the constituents and opacity of plasma are different, so that plasma of different samples will shield postpulse and subsequently ablate the sample surface with different efficiencies and to different extents. Therefore, the spectral line intensity of the same element in different samples would exhibit an inconsistent relationship with respect to elemental concentrations. But for fs laser pulse, before the plasma formation and other thermal response, the interaction between the laser and matter has finished and all the laser energy has already been deposited on the sample surface. There are no further effects or processes that impact the plasma formation. Power density and electron density are very high inside the fs filaments, and especially higher in the fs plasma grating so that the sample pellets could be ablated. As a result, FIBS and especially GIBS could overcome the matrix effect effectively.

\section{Conclusions}

We used a plasma grating from the interference of two noncollinear fs filaments to ablate and detect sample pellets. The interaction of two filaments leads to more intense electron acceleration and collision so that the local electron density inside the plasma grating is higher than that obtained with a single fs filament. The signal intensity of spectral lines detected by GIBS is enhanced by more than 3 times that obtained from a single FIBS system. The lifetime of grating-induced plasma is apparently extended. Finally, we demonstrate that using the GIBS technique, materials could be ionized and dissociated, effectively leading to reduction of the matrix effect. The GIBS technique could be a promising tool to detect samples hard to melt, ionize, or dissociate, and samples with a complex matrix.

\section{Acknowledgments}

We acknowledge the support from the National Key Research and Development Program (No. 2018YFB0407100), the National Natural Science Foundation of China (No. 11621404), and the Key Project of Shanghai Education Commission (No. 201701-07-00-05-E00021).

\section{References}

1. C. López-Moreno et al., "Test of a stand-off laser-induced breakdown spectroscopy sensor for the detection of explosive residues on solid surfaces," J. Anal. At. Spectrom. 21(1), 55-60 (2006).
2. D. W. Hahn and N. Omenetto, "Laser-induced breakdown spectroscopy (LIBS), part II: review of instrumental and methodological approaches to material analysis and applications to different fields," Appl. Spectrosc. 66(4), 347-419 (2012).

3. A. K. Shaik et al., "Femtosecond laser induced breakdown spectroscopy based standoff detection of explosives and discrimination using principal component analysis," Opt. Express 26(7), 80698083 (2018).

4. A. K. Shaik, Ajmathulla, and V. R. Soma, "Discrimination of bimetallic alloy targets using femtosecond filament-induced breakdown spectroscopy in standoff mode," Opt. Lett. 43(15), 3465-3468 (2018).

5. G. Arca et al., "Trace element analysis in water by the laserinduced breakdown spectroscopy technique," Appl. Spectrosc. 51(8), 1102-1105 (1997).

6. V. Lazic and S. Jovićević, "Laser induced breakdown spectroscopy inside liquids: processes and analytical aspects," Spectrochim. Acta, Part B 101, 288-311 (2014).

7. Y. Tian et al., "Non-gated laser-induced breakdown spectroscopy in bulk water by position-selective detection," Appl. Phys. Lett. 107, 111107 (2015).

8. S. Niu et al., "Laser-induced breakdown spectroscopic detection of trace level heavy metal in solutions on a laser-pretreated metallic target," Talanta 179, 312-317 (2018).

9. C. Haisch et al., "Element-specific determination of chlorine in gases by laser-induced-breakdown-spectroscopy (LIBS)," Fresenius J. Anal. Chem. 356, 21-26 (1996).

10. V. Sturm and R. Noll, "Laser-induced breakdown spectroscopy of gas mixtures of air, $\mathrm{CO}_{2}, \mathrm{~N}_{2}$, and $\mathrm{C}_{3} \mathrm{H}_{8}$ for simultaneous $\mathrm{C}, \mathrm{H}, \mathrm{O}$, and N measurement," Appl. Opt. 42(30), 6221-6225 (2003).

11. K. L. Eland et al., "Energy dependence of emission intensity and temperature in a LIBS plasma using femtosecond excitation," Appl. Spectrosc. 55(3), 286-291 (2001).

12. B. N. Chichkov et al., "Femtosecond, picosecond and nanosecond laser ablation of solids," Appl. Phys. A 63(2), 109-115 (1996).

13. V. Margetic et al., "A comparison of nanosecond and femtosecond laser-induced plasma spectroscopy of brass samples," Spectrochim. Acta, Part B 55(11), 1771-1785 (2000).

14. D. J. Hwang et al., "Femtosecond laser ablation induced plasma characteristics from submicron craters in thin metal film," Appl. Phys. Lett. 91(25), 251118 (2007).

15. G. G. Arantes de Carvalho et al., "Direct determination of the nutrient profile in plant materials by femtosecond laser-induced breakdown spectroscopy," Anal. Chim. Acta 876, 26-38 (2015).

16. H. L. Xu et al., "Femtosecond laser-induced nonlinear spectroscopy for remote sensing of methane," Appl. Phys. B 82(4), 655-658 (2006).

17. K. Stelmaszczyk et al., "Long-distance remote laser-induced breakdown spectroscopy using filamentation in air," Appl. Phys. Lett. 85(18), 3977-3979 (2004).

18. A. Couairon and A. Mysyrowicz, "Femtosecond filamentation in transparent media," Phys. Rep. 441(2-4), 47-189 (2007).

19. S. L. Chin et al., "Advances in intense femtosecond laser filamentation in air," Laser Phys. 22(1), 1-53 (2012).

20. A. Braun et al., "Self-channeling of high-peak-power femtosecond laser pulses in air," Opt. Lett. 20(1), 73-75 (1995).

21. S. S. Harilal et al., "Dynamics of molecular emission features from nanosecond, femtosecond laser and filament ablation plasmas," J. Anal. At. Spectrom. 31(6), 1192-1197 (2016).

22. S. S. Harilal et al., "Consequences of femtosecond laser filament generation conditions in standoff laser induced breakdown spectroscopy," Opt. Express 24(16), 17941-17949 (2016).

23. F. Théberge et al., "Plasma density inside a femtosecond laser filament in air: strong dependence on external focusing," Phys. Rev. E 74(3), 036406 (2006).

24. S. Eisenmann, A. Pukhov, and A. Zigler, "Fine structure of a laser-plasma filament in air," Phys. Rev. Lett. 98(15), 155002 (2007). 
25. Y. H. Chen et al., "Direct measurement of the electron density of extended femtosecond laser pulse-induced filaments," Phys. Rev. Lett. 105(21), 215005 (2010).

26. P. P. Kiran et al., "Filamentation without intensity clamping," Opt. Express 18(20), 21504-21510 (2010).

27. $\mathrm{H}$. Wu et al., "Background-free third-order harmonic generation induced by dynamic gratings in dual filaments," J. Opt. Soc. Am. B 26(4), 645-649 (2009).

28. X. Yang et al., "Plasma waveguide array induced by filament interaction," Opt. Lett. 34(24), 3806-3808 (2009).

29. X. Yang et al., "Femtosecond laser pulse energy transfer induced by plasma grating due to filament interaction in air," Appl. Phys. Lett. 97(7), 071108 (2010).

30. S. Suntsov et al., "Femtosecond laser induced plasma diffraction gratings in air as photonic devices for high intensity laser applications," Appl. Phys. Lett. 94(25), 251104 (2009).

31. L. Shi et al., "Generation of high-density electrons based on plasma grating induced Bragg diffraction in air," Phys. Rev. Lett. 107(9), 095004 (2011).

32. A. C. Bernstein et al., "Two-beam coupling between filamentforming beams in air," Phys. Rev. Lett. 102(12), 123902 (2009).

33. C. $\mathrm{Ng}$ et al., "Spectrochemical analysis of liquids using laserinduced plasma emissions: effects of laser wavelength on plasma properties," Appl. Spectrosc. 51(7), 976-983 (1997).

34. L. Zheng et al., "Comparative study of the matrix effect in $\mathrm{Cl}$ analysis with laser-induced breakdown spectroscopy in a pellet or in a dried solution layer on a metallic target," Spectrochim. Acta, Part B 118, 66-71 (2016).
Mengyun Hu received her MS degree in environmental engineering from the East China Normal University in 2014. In the same year, she joined the State Key Laboratory of Precision Spectroscopy of the East China Normal University, as an engineer. She has participated in the National Key Research and Development Program of China. Her current research interests are in laser applications.

Junsong Peng received his $\mathrm{PhD}$ in optical engineering from Shanghai Jiao Tong University in 2003 and did his research at the Royal Swedish Institute of Technology and as the Marie Curie Fellow of Aston University, United Kingdom, successively. Since 2017, he has been at the State Key Laboratory of Precision Spectroscopy of the East China Normal University. His research focuses on femtosecond laser dynamics, optical solitons, and nonlinear fiber optics.

Sheng Niu received his $\mathrm{PhD}$ in optics, supervised by Prof. Heping Zeng, from East China Normal University in 2019. His main research interest is laser-induced breakdown spectroscopy.

Heping Zeng received his PhD from Shanghai Institute of Optics and Fine Mechanics at the Chinese Academy of Sciences. He is a professor of the State Key Laboratory of Precision Spectroscopy, East China Normal University, Shanghai, China. He was selected as a distinguished young scholar of NSFC. He was invited to serve as an editorial board member for European Physical Journal D. He is a fellow of OSA. His research focuses on precision control of the light field and its application in precision spectroscopy. 